\title{
Terapia nutricional em pacientes com Doença Renal Crônica: Revisão narrativa
}

\author{
Nutritional therapy in patients with Chronic Kidney Disease: Narrative review \\ Terapia nutricional en pacientes con Enfermedad Renal Crónica: Revisión narrativa
}

Recebido: 09/03/2021 | Revisado: 16/03/2021 | Aceito: 23/03/2021 | Publicado: 31/03/2021

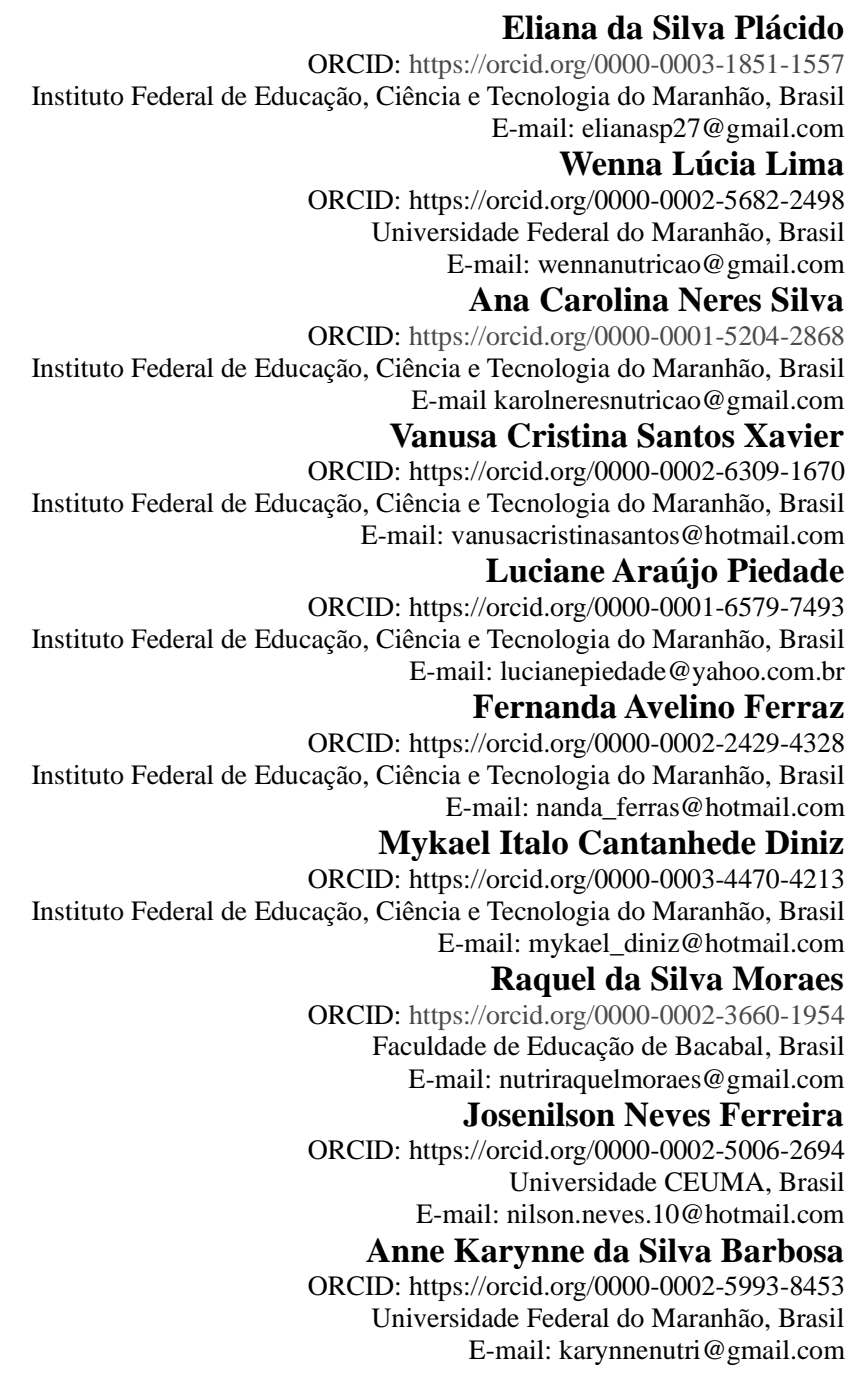

\section{Resumo}

Pesquisar sobre a terapia nutricional na doença renal crônica. Trata-se de uma revisão de literatura de caráter integrativo, através de busca sistematizada com os termos descritos no DeSC (Terapia Nutricional, Assistência ao paciente, Insuficiência renal crônica) através da combinação entre eles, usando os operadores booleanos (AND, OR, AND NOT), essa busca foi realizada através de uma vasta pesquisa com estudos dos últimos 19 anos, nas bases de dados Pubmed, Scielo e Periódico Capes, foram utilizados todos os textos que foram achados relevantes para o estudo, foram excluídos ao artigos que não eram de acesso aberto e os que não versavam sobre a temática. A filtração renal é a principal e mais conhecida função dos rins sendo realizada pelo processo de ultrafiltração e reabsorção glomerular. A ultrafiltração ocorre com a transferência seletiva de íons, moléculas e água através dos capilares que formam o glomérulo, num espaço do néfron conhecido por cápsula de Bowman, em contrapartida a reabsorção decorre da passagem de substâncias para o lado de fora do túbulo do néfron, do interstício ou para os capilares renais. De acordo com o estudo foi observado, que os portadores de doença renal crônica em hemodiálise são um público vulnerável, sendo necessário o constante acompanhamento do estado nutricional por ser um forte preditor de morbidades e mortalidades.

Palavras-chave: Terapia nutricional; Assistência ao paciente; Insuficiência renal crônica. 


\begin{abstract}
Research on nutritional therapy in chronic kidney disease. This is an integrative literature review, through a systematic search with the terms described in the DeSC (Nutritional Therapy, Patient Care, Chronic Kidney Failure) through the combination of them, using the Boolean operators (AND, OR, AND NOT), this search was carried out through a vast research with studies from the last 19 years, in the databases Pubmed, Scielo and Periódico Capes, all texts that were found relevant to the study were used, were excluded from the articles that they were not open access and those that did not deal with the theme. Renal filtration is the main and best-known function of the kidneys, being carried out by the process of ultrafiltration and glomerular reabsorption. Ultrafiltration occurs with the selective transfer of ions, molecules and water through the capillaries that form the glomerulus, in a space in the nephron known as the Bowman's capsule, in contrast the reabsorption results from the passage of substances outside the nephron tubule, of the interstice or to the renal capillaries. According to the study, it was observed that patients with chronic kidney disease on hemodialysis are a vulnerable public, requiring constant monitoring of nutritional status as it is a strong predictor of morbidities and mortality.
\end{abstract}

Keywords: Nutritional therapy; Patient care; Chronic renal failure.

\title{
Resumen
}

Investigación sobre terapia nutricional en enfermedad renal crónica. Se trata de una revisión integradora de la literatura, mediante una búsqueda sistemática con los términos descritos en la DeSC (Nutritional Therapy, Patient Care, Chronic Kidney Failure) mediante la combinación de los mismos, utilizando los operadores booleanos (AND, OR , Y NO), esta búsqueda se realizó a través de una amplia investigación con estudios de los últimos 19 años, en las bases de datos Pubmed, Scielo y Periódico Capes, se utilizaron todos los textos que se encontraron relevantes para el estudio, fueron excluidos de los artículos que no eran de acceso abierto y los que no abordaban el tema. La filtración renal es la función principal y más conocida de los riñones que se realiza mediante el proceso de ultrafiltración y reabsorción glomerular. La ultrafiltración ocurre con la transferencia selectiva de iones, moléculas y agua a través de los capilares que forman el glomérulo, en un espacio de la nefrona conocido como cápsula de Bowman, en contraste la reabsorción resulta del paso de sustancias fuera del túbulo de la nefrona, del intersticio o de los capilares renales. Según el estudio, se observó que los pacientes con enfermedad renal crónica en hemodiálisis son un público vulnerable, requiriendo un monitoreo constante del estado nutricional ya que es un fuerte predictor de morbilidad y mortalidad.

Palabras clave: Terapia nutricional; Atención al paciente; Insuficiencia renal crónica.

\section{Introdução}

A doença renal crônica é a alteração progressiva e irreversível da capacidade funcional dos rins, mediada inicialmente por lesão e que posteriormente dificulta o processo normal de filtração (Santos et al., 2013). Nesse sentido, o indivíduo que apresenta taxa de filtração glomerular (TFG) “ $<60 \mathrm{ml} / \mathrm{min} / 1,73 \mathrm{~m}^{2}$ associada ou não a alguma patologia é diagnosticada com a Doença Renal Crônica (DRC)” (Bastos; Kirsztajn, 2011).

Dessa forma, a gravidade da doença é medida de acordo com a taxa e capacidade de filtração glomerular, onde o estágio varia de 0 a 6 com progressão da lesão, redução da filtração e consequente perda da função à medida que os estágios evoluem. No último estágio os rins possuem capacidade de filtração glomerular $<15 \mathrm{ml} / \mathrm{min} / 1,73 \mathrm{~m}^{2}$ caracterizando o quadro de "insuficiência renal terminal ou dialítica" (Brasil, 2006).

A lesão renal pode ser desencadeada por diversos fatores de riscos, estes, segundo Siviero; Rodrigues e Machado (2013) são classificados como grupo das doenças de base, dos quais fazem parte hipertensão, seguida de diabetes e glomerulonefrites (Cherchiglia et al., 2010). A "História familiar, doença renal policística, doenças autoimunes, infecções sistêmicas, infecções urinárias de repetição, litíase urinária, uropatias obstrutivas e neoplasias," compõem o outro grupo de fatores relacionados a perda da funcionalidade renal e ao surgimento da doença (Brasil, 2006).

A doença renal crônica abrange níveis altamente prevalentes no mundo. No Brasil a prevalência é estabelecida com base na quantidade de pessoas que são submetidas ao Tratamento de Substituição Renal (TSR), sendo, estes: "tratamento dialítico [hemodiálise (HD)], diálise peritoneal e transplante renal" (Moura et al., 2009). De acordo com os resultados do Censo Brasileiro de Diálise Crônica realizado pela Sociedade Brasileira de Nefrologia (SBN) o número total de doentes renais crônicos em processo de diálise no ano de 2013 foi de “100.397” (Sesso et al., 2014). 
De acordo com Siviero; Rodrigues e Machado (2013) a DRC acomete pessoas de qualquer idade, sendo esta, caracterizada como um dos fatores de riscos secundários essa patologia. Dessa forma, pessoas com idade >60 anos principalmente do sexo masculino enquadram-se nesses fatores e são mais propensos ao desenvolvimento dessa patologia. Entende-se, portanto, que pela alta prevalência e incidência refletidas nas taxas de morbidade, tal doença é destacada como um relevante problema de saúde pública.

A ausência da funcionalidade renal promove ao organismo alterações orgânicas generalizadas devido a disfunção do seu potencial de excreção, síntese e de regulação. Concomitantemente ao tratamento médico o paciente deve fazer acompanhamento dietético, pois exige-se alterações no consumo alimentar, devido as condições impostas pela própria doença (National Kidney Foundation, 2007).

O fator dietético do paciente renal é um delicado processo determinante da qualidade de vida, importante para a resposta ao tratamento e preservação da vida do doente. Mahan e Scott-Stump (2011) abordam que é comum a ocorrência de inapetência principalmente nos que são submetidos aos primeiros processos dialíticos, portanto, para a prevenção de alterações nutricionais do paciente é indispensável que este, siga fidedignamente as prescrições dietéticas e orientações nutricionais, principalmente em relação ao consumo de proteína, fósforo e potássio que estão envolvidos com algumas das principais complicações referentes à doença, ou seja, desnutrição e mortalidade respectivamente.

Nessa abordagem observou-se a necessidade de monitorar o consumo alimentar e o estado nutricional dos pacientes afim de evitar possíveis complicações.

\section{Metodologia}

Trata-se de uma revisão de literatura de caráter integrativo, através de busca sistematizada com os termos descritos no DeSC (Terapia Nutricional, Assistência ao paciente, Insuficiência renal crônica) através da combinação entre eles, usando os operadores booleanos (AND, OR, AND NOT), essa busca foi realizada através de uma vasta pesquisa com estudos dos últimos 19 anos, nas bases de dados Pubmed, Scielo e Periódico Capes, foram utilizados todos os textos que foram achados relevantes para o estudo, foram excluídos ao artigos que não eram de acesso aberto e os que não versavam sobre a temática.

\section{Resultados e Discussão}

\section{Função dos rins}

A filtração renal é a principal e mais conhecida função dos rins sendo realizada pelo processo de ultrafiltração e reabsorção glomerular (Sesso et al., 2014). A ultrafiltração ocorre com a transferência seletiva de íons, moléculas e água através dos capilares que formam o glomérulo, num espaço do néfron conhecido por capsula de Bowman, em contrapartida a reabsorção decorre da passagem de substâncias para o lado de fora do túbulo do néfron, do interstício ou para os capilares renais (Santos et al., 2013). Por meio desses dois fatores é possível entender que os rins, através de suas unidades funcionais altamente especializadas, realizam um meticuloso processo de purificação, conseguindo eliminar componentes prejudiciais em quantidades importantes e reabsorver substâncias ainda necessárias ao organismo humano (Motta, 2009).

Além de exercer sua função principal de filtração e reabsorção glomerular de eletrólitos, líquidos e solutos orgânicos, os rins também realizam funções como, eliminação de substâncias metabólicas e substratos químicos indesejáveis (Caldas, 2011), ajuste no balanço de água e eletrólitos promovendo a homeostase do meio interno, ajuste nos níveis pressóricos, atua na liberação, metabolização e eliminação de hormônios, bem como na gliconeogênese e no equilíbrio acidobásico (Hall, 2011).

Os produtos finais do metabolismo dos quais são primariamente eliminados pelos rins são: os ácidos, sendo os mais comuns o úrico e os orgânicos: lático, ascórbico e acético, drogas e possíveis toxinas formadas por elas, produtos do metabolismo proteico e muscular: uréia e creatinina e produtos de degradação da hemoglobina como a bilirrubina (Motta, 
2009), estes devem ser eliminados rapidamente do meio interno corporal pela inutilidade ao organismo e pelo perigo que causam a saúde se estiverem presentes em grandes quantidades (Caldas, 2011).

Quanto a função do equilíbrio de água e eletrólitos os rins realizam o controle de acordo com a demanda, ou seja, a eliminação de água ocorre com base no ganho, dessa forma se o consumo ultrapassar a quantidade a ser eliminada, o volume de água e eletrólitos irá aumentar, do contrário, se o ganho de água for menor em relação a quantidade excretada a tendência é que o volume de água e eletrólitos diminua (Hall, 2011).

Outra função atribuída aos rins relaciona-se ao controle da pressão sanguínea que ocorre tanto pela regulação dos níveis de água e sódio quanto pela liberação de produtos vasoconstrictores que atuam sobre os tecidos periféricos através de um mecanismo denominado renina-angiotensina (Motta, 2009), este "equivale ao maior controle da pressão, onde a renina, enzima proteolítica secretada pelos glomérulos atua sobre o angiotensinogênio no plasma para formar angiotensina I que é posteriormente convertida em angiotensina II" (Hall, 2011), substância vasoativa que atua estimulando o hormônio aldosterona responsável por manter os níveis normais da pressão arterial através do controle do volume sanguíneo pela reabsorção de água e sódio (Mahan; Escott-Stump 2011).

Os rins como órgãos endócrinos realizam a síntese da eritropoietina, um hormônio responsável por estimular a medula óssea a produzir as células vermelhas. A secreção diminuída de eritropoietina promove o desenvolvimento da doença renal e o consequente decréscimo do trabalho da medula óssea, propiciando o surgimento da anemia nos doentes renais (Eaton; Pooler 2005).

Apesar de o fígado ser o principal órgão responsável pelo processo de gliconeogênese, os rins num longo período de jejum, também possuem o papel de realizar a síntese de glicose por meio de aminoácidos e outros precursores (Hall, 2011). A atividade renal também está ligada a produção da 1,25-di-hidroxivitamina $\mathrm{D} 3$, a forma ativa da vitamina $\mathrm{D}$, que proporciona o equilíbrio homeostático do cálcio e fósforo, facilitando a nível intestinal o processo absortivo do cálcio, um componente importante para o remodelamento e manutenção óssea (Mahan; Escott-Stump, 2011).

O balanço acidobásico é uma das atividades mais importantes dos rins, através deste, os ácidos corporais advindos do metabolismo tais como: "ácidos sulfúrico, fosfórico, clorídrico, pirúvico, láctico e cítrico além de corpos cetônicos" (Motta, 2009) são eliminados do corpo prevenindo a ocorrência de um evento conhecido como acidose metabólica do qual pode prejudicar a perfeita funcionalidade de tecidos e órgãos, com ênfase nos músculos e ossos, favorecendo o hipercatabolismo muscular, desnutrição e aumento dos índices de mortalidade (SBN, 2011).

Toda a funcionalidade renal torna-se alterada quando os rins são acometidos por alguma lesão, que pode progredir ou não para processos patológicos. De acordo com Hall (2011), com a lesão renal, manifestação de doenças e/ou processos fisiológicos do envelhecimento, acontece a redução progressiva da quantidade de néfrons, porém apesar desse declínio, segundo o referido autor não há risco de vida, visto que os rins possuem alta capacidade adaptativa (Motta, 2009). Por outro lado, se a lesão se tornar persistente todo o processo funcional de equilíbrio, controle endócrino, pressórico dentre outros, tornam-se alterados, sendo a filtração a função mais acometida. Um dos aspectos observados na filtração desregulada, referentes ao processo de excreção renal é chamado de azotemia, que ocorre quando os "produtos normais de excreção não são eliminados de maneira apropriada e reúnem-se em quantidades anormais no sangue" (Mahan; Escott-Stump, 2011).

Quando a função renal não ocorre de maneira adequada, ou seja, quando os rins são incapazes de segregar a quantidade diária de produtos, dá-se o que se chama de insuficiência renal que pode se ocasionar de duas formas: aguda e crônica (Motta, 2009). Percebe-se que o período de ocorrência é o que difere uma da outra, visto que, na insuficiência aguda a funcionalidade reduz abruptamente num período que dura, horas ou dias (Hall, 2011), onde o ritmo de filtração glomerular torna-se cada vez mais vagaroso e reflete um volume urinário diminuído, além disso, ocorrem falhas no controle acidobásico e 
hidroeletrolítico que se mantém cada vez menos em equilíbrio, já a insuficiência crônica se desenvolve em um período de filtração lento e constante (SBN, 2007).

\section{Doença renal crônica}

$\mathrm{Na}$ insuficiência renal crônica o número de néfrons diminui de forma progressiva e irreversível em decorrência de processos fisiológicos, relacionados à idade e de processos patológicos que promovem destruição tecidual, também conhecida por lesão parenquimatosa renal. As lesões que promovem o estabelecimento e desenvolvimento da patologia podem surgir por consequência de diversas doenças como "diabetes melito, obesidade, hipertensão, glomerulonefrite e cálculos renais" (Hall, 2011).

Esses fatores causam alteração nos constituintes renais, redução do número de néfrons e determinam a perda da capacidade funcional renal, tanto exócrina, como a filtração, que pela atividade diminuída promove o acúmulo de água, eletrólitos e compostos metabólicos como a uréia, (Romão Junior, 2004). Quanto às endócrinas relacionadas à produção diminuída de 1,25-didroxivitamina D3 e do hormônio eritropoietina (Chaves; Graça; Gallo, 2007).

A infuncionalidade renal é, portanto, fator determinante para a ocorrência da Doença Renal em Estágio Terminal, estabelecida quando os rins possuem um número total de néfrons que varia de 70\% a 75\% abaixo do normal, refletindo taxa de filtração $<15 \mathrm{ml} / \mathrm{min} / 1,73 \mathrm{~m}^{2}$ (Hall, 2011).

A sequência do processo fisiopatológico da Doença Renal em Estágio Terminal ocorre primeiramente com o decréscimo da quantidade de néfrons funcionais, em fator disso, há elevação expressiva do volume de soluto por néfron, proporcionando a desregulação na taxa de filtração e diminuição dos glomérulos de reserva; os rins tornam-se insuficientes e por fim ocorre a instauração da doença renal terminal associada ao definhamento fibroso nos túbulos renais (Antczak, 2005).

Quando a doença renal terminal se instala entende-se que ocorre a quebra de um ciclo vicioso de adaptação renal, onde os néfrons sobreviventes se hipertrofiam afim, de exercer a função dos que não resistiram à lesão, dessa forma a filtração glomerular fica compensada (Hall, 2011). As mudanças adaptativas tornam possível a eliminação de valores adequados de água e outras substâncias mesmo com a redução de cerca de $20 \%$ a $25 \%$ do volume funcional renal (Sesso et al., 2013). Entretanto com o passar dos anos, tais mudanças concorrem para o estabelecimento da lesão nefrótica adicional, ou seja, os glomérulos hiperfiltrantes, "tendem a se deteriorar em médio ou longo prazo" (Zambra; Huth, 2010).

A partir do momento em que a capacidade adaptativa dos rins não mais consegue ser exercida, ou seja, quando a doença já encontra-se no estágio terminal a sintomatologia que antes se fazia ou não presente nos estágios, começa definitivamente a se manifestar e o paciente pode apresentar nessa fase mudanças na aparência da urina, dor ou ardência ao urinar, inchaço nos tornozelos e nas pálpebras, além de fraqueza, tonturas e alterações na elevação da pressão (Moura, 2014).

Cerca de 90\% dos portadores de doença renal em estágio terminal possuem hipertensão, diabetes ou glomerulonefrites (Mahan; Escott-Stump, 2011), sendo a hipertensão a que possui maior incidência nos pacientes renais, esta tende a ocorrer em mais de 75\% dos doentes de qualquer faixa etária (Bastos; Bregman, Kirsztajn, 2010).

A hipertensão pode assumir dentro da doença renal crônica, duas vertentes, nesse sentido, entende-se que a mesma pode tanto ser uma das causas da doença, por conta do agravo da lesão que promove nos vasos e capilares renais, quanto ser desencadeada pela doença por conta da desregulação da função renal, dessa forma, a hipertensão pode tanto causar a doença como a própria doença pode causar a hipertensão (Hall, 2011).

Frente aos aspectos abordados, indaga-se o porquê da hipertensão arterial ser considerada a principal doença de base para o desenvolvimento progressivo da patologia renal, a partir dessa pauta, entende-se que não só o fator lesão, mas vários outros estão envolvidos em todo esse processo, um deles refere-se ao fato de que a maioria dos portadores da doença, os hipertensos (Moura, 2014), desconhecem que estão doentes, além disso, "entre aqueles que conhecem o diagnóstico, apenas 
20\% são adequadamente tratados. Nesse sentido, sem identificação e tratamento adequado, há grandes chances da hipertensão manter seu papel como importante causa de Doença Renal Crônica Terminal” (Sesso; Gordan, 2007).

De acordo com a Sociedade Brasileira de Diabetes a nefropatia diabética provoca a disfunção renal por conta do longo período de exposição dos capilares à hiperglicemia, aliada ao péssimo controle pressórico sanguíneo, a colesterolemia, ao tabagismo e a fatores genéticos (Sá, 2014).

Para Moreira et al., (2008) cerca de 30\% a 40\% dos diabéticos tipo 1 e $10 \%$ a $40 \%$ dos diabéticos tipo 2 são portadores de nefropatia diabética. Sesso e Gordan (2007) abordam que o número de diabéticos acometidos com nefropatia nos últimos anos é crescente, porém, muitos deles acabam morrendo de outras complicações antes de chegarem ao estágio terminal da doença renal.

Tal como o nome sugere as glomerulonefrites representam um conjunto de doenças inflamatórias intimamente relacionadas com o seguimento da doença renal em estágio terminal, estas podem se apresentar na forma aguda e crônica, e ser ou não submetidas ao tratamento (Siviero; Machado; Rodrigues, 2013).

A doença renal é identificada pelo grau de funcionalidade renal independente do diagnóstico apresentado pelo paciente (Romão Junior, 2004), a classificação do grau de insuficiência dá-se através de seis estágios. O primeiro, denominado de estágio 0 , caracteriza-se por filtração glomerular $>90\left(\mathrm{ml} / \mathrm{min} / 1,73 \mathrm{~m}^{2}\right)$, determinando a inexistência de lesões renais. Nesse estágio estão incluídos os idosos, diabéticos, hipertensos, os que ainda não apresentam a lesão renal, mas que possuem relação hereditária com o processo patológico e todos aqueles que fazem parte do chamado grupo de risco para o desenvolvimento da doença (SBN, 2007).

No estágio 1 a filtração também se encontra $>90\left(\mathrm{ml} / \mathrm{min} / 1,73 \mathrm{~m}^{2}\right)$, porém a lesão renal está em estágio inicial, o que já favorece a perda de proteína e albumina do meio interno. É importante frisar que em nenhum dos dois primeiros estágios é constatado a ocorrência de disfunção renal.

A taxa de filtração no estágio 2 encontra-se diminuída, visto que os rins filtram de 60 a $89\left(\mathrm{ml} / \mathrm{min} / 1,73 \mathrm{~m}^{2}\right)$. A insuficiência renal nesse estágio é presente e caracterizada como leve e apesar disso as concentrações de uréia e creatinina encontram-se normais, clinicamente o paciente não apresenta sinais e sintomas dignos de importância na insuficiência e somente métodos precisos de avaliação da função renal irão apontar tais anormalidades (Romão Junior, 2004).

A lesão renal encontra-se totalmente instalada no estágio 3 da doença e o processo de filtração ainda mais diminuído, variando de 30 a $59\left(\mathrm{ml} / \mathrm{min} / 1,73 \mathrm{~m}^{2}\right)$ refletindo de acordo com Brasil (2006) funcionamento renal moderado. Os sinais e sintomas apresentam-se de forma amena, ligados principalmente aos fatores desencadeadores como infecções urinárias, hipertensão, diabetes melitus e lúpus (Romão Junior, 2007).

A insuficiência renal no estágio 4 descrita como grave ou severa, possui taxa de filtração glomerular de 15 a 29 $\mathrm{ml} / \mathrm{min}$. A disfunção renal promove a presença de sinais e sintomas típicos de uremia como ânsia acompanhada de vômitos, perda da capacidade gustativa que refletem no desejo de comer, emagrecimento, edemaciamento, palidez, falta de ar (SBN, 2007).

Quando a filtração glomerular se encontra menos que $15 \mathrm{ml} / \mathrm{mim}$ entende-se que o paciente se encontra no estágio terminal da doença. O rim é incapaz de adaptar-se e controlar suas funções, para tanto, torna-se totalmente indispensável à vida o controle dos sintomas, dos quais são intensamente presentes nessa fase, como solução o paciente deve submeter-se ao tratamento de substituição renal, realizando o processo de "depuração artificial do sangue (diálise peritoneal ou hemodiálise) ou o transplante renal" (Romão Junior, 2004). 


\section{Hemodiálise como terapia de substituição renal}

Segundo Sostisso, (2011) a perda da função renal que ocorre de forma lenta, contínua e irreversível, faz com que o portador da doença, necessite de auxílio para a execução das funções antes realizadas pelos rins. As terapias de substituição renal são, portanto, o meio de sobrevivência e prolongamento da vida nos pacientes renais crônicos. As principais formas de tratamento são diálise peritoneal, hemodiálise e transplante renal, sendo a hemodiálise uma das mais comuns formas de tratamento renal realizada no Brasil com quantidade estimada de 100.397 pacientes no ano de 2013 (Sesso et al., 2014).

O tratamento ocorre durante 3 vezes na semana num período que varia de 3 a 5 horas, onde profissionais devidamente treinados prestam cuidados a todo momento (Hiae, 2014).

Nascimento; Marques (2005), descrevem o processo de diálise como um método de filtragem e purificação do sangue de elementos não "suportáveis como creatinina e a uréia" que devem ser excretadas do organismo. A hemodiálise funciona com a captação do sangue do paciente pela máquina (dialisador), através de um acesso venoso, um cateter ou fistulas arteriovenosas, o sangue do paciente é impulsionado com auxílio de uma bomba até o filtro dialisador onde o mesmo será exposto a uma solução da diálise por meio de uma membrana responsável por separar as partes liquidas e substancias toxicas ao organismo fazendo voltar o sangue purificado através da entrada da fístula aberta no organismo do paciente (SBN, 2014).

Canto, (2009) conclui que a diálise é um método que proporciona uma maior sobrevida aos pacientes portadores de insuficiência renal crônica, no entanto está longe de suprir todas as funções desempenhadas pelo rim normal, sobretudo na sua função endócrina, metabólica e de produção.

O tratamento dialítico provoca alterações físicas e emocionais nos pacientes, mudanças essas que refletem também na alimentação, que por vezes é alterada, suscitando a modificações no estado nutricional do indivíduo (Mahan; Scott-Stump, 2011). Valenzuela et al., (2003), cita que alterações hormonais, como síntese ineficiente de hormônios importantes como a eritropoietina, alterações principalmente relacionadas ao metabolismo proteico e energético e mudanças na ingestão alimentar, provocadas principalmente por vômitos náuseas e ocorrências do estado de toxicidade do organismo, são causas de alterações do estado nutricional nos pacientes. Pesquisas estão sendo desenvolvidas, sobre a associação da desnutrição proteico calórica em doentes renais, relacionando as causas e efeitos pelo fato da sua elevada "prevalência" e estreita relação com o aumento da "mortalidade" (Kamimura et al., 2004).

Funchs, (2011), salienta que o tratamento dialítico como dito anteriormente por si só traz transtornos ao indivíduo, o estado de enfermidade, o estresse causado pela doença, promove o aumento do catabolismo proteico e energético suscitando a perda de aminoácidos e peptídeos bem como de vitaminas hidrossolúveis.

Canto, (2009) afirma que 6 a $8 \%$ dos pacientes em tratamento hemodialítico padecem de desnutrição grave, e por volta de $33 \%$ de desnutrição leve a moderada. O processo de hemodiálise promove ainda intercorrências agudas durante ou após o processo dialítico, como cefaleia, calafrios, delírios, confusão mental, convulsões, náuseas e vômitos, hipoxemia, arritmias cardíacas, alergias e prurido, no entanto a hipotensão arterial e as câimbras musculares são descritas como as ocorrências mais comumente apresentadas, representando um percentual de 20\% de acordo com estudos de Castro (2001).

Ocorrem complicações quando o paciente possui uma quantidade excessiva de líquido retido em seu corpo e para que estas sejam evitadas é importante que o mesmo siga corretamente a prescrição dietética, faça uso correto de medicamentos e observe com atenção as orientações médicas, para que assim, possa vir a ter uma sessão dialítica satisfatória (SBN, 2014).

\section{Estado Nutricional do Paciente Renal Crônico}

A desnutrição, complicação comum nos doentes renais, acomete $10 \%$ a $70 \%$ dos pacientes que realizam tratamento hemodialítico e 18\% a 56\% dos que são tratados pela diálise peritoneal (Barbosa; Andrade Junior; Bastos, 2007). 
O desequilíbrio hormonal e gastrointestinal, as perdas de nutrientes decorrentes do processo dialítico a limitação a certos alimentos, o uso de medicamentos e a uremia, desencadeiam episódios de náuseas, vômitos e anorexia e promovem alterações no estado nutricional do paciente (Cabral; Diniz; Arruda, 2005).

A inadequação de peso e as baixas concentrações séricas de colesterol e albumina aumentam o risco de morbimortalidade nos pacientes. (Barbosa; Andrade Junior; Bastos, 2007). O excesso de peso quando determinado pelo IMC em doentes renais, pode reduzir as complicações associadas e os riscos de morte, porém, essa vantagem só é válida quando a massa magra do indivíduo estiver adequada, do contrário, essa proteção pode auxiliar o desenvolvimento de alterações metabólicas e promover a mortalidade nos pacientes terminais (Freitas et al., 2013).

A desnutrição tende a prevalecer e agravar-se, com base nos anos de diálise realizados pelo paciente, sendo, portanto, mais recorrentes nos idosos. Através de uma alimentação adequada é possível prevenir o definhamento do indivíduo, dessa forma, a via oral quando estimulada e acompanhada se necessário de suplementação demonstra ser eficiente e bem aceita para manutenção do peso e prevenção de complicações (SBNPE, 2011).

Os métodos mais comuns na prática clínica para determinar o estado nutricional do paciente renal crônico, são os chamados métodos objetivos, que se caracterizam pelos índices antropométricos de peso e altura, IMC, aferição de pregas cutâneas, como a dobra cutânea tricipital (DCT), circunferência do braço (CB) e circunferência muscular do braço (CMB), sendo considerados métodos simples e objetivos para avaliar as reservas corporais dos indivíduos, possuindo uma boa correlação com os parâmetros bioquímicos (Kamimura; AVESSANI; CUPARI, 2003).

\section{Terapia nutricional do paciente renal crônico em hemodiálise}

A dieta segundo a National Kidney Foundation, (2007) possui parcela significativa no tratamento de doentes renais crônicos, principalmente no que se refere à prevenção de certas complicações clínicas. Estudos abordam a desnutrição proteicoenergética como o distúrbio patológico mais frequentemente relacionado à doença, compreendendo um percentual de cerca de 20 a $80 \%$ de manifestação nos pacientes que realizam hemodiálise, esse mal que altera por completo o estado de saúde do paciente, resulta basicamente de processos anorexígenos relacionados ao mecanismo de regulação do apetite e saciedade (Oliveira et al., 2010).

As causas da anorexia descritas por Fuchs, (2011) podem ser destacadas a carga urêmica sanguínea, que em altas concentrações pode desencadear processos de toxidez no organismo, uso demasiado de medicamentos que acabam por intervir no processo alimentar, principalmente pela alteração no paladar, fatores psicoemocionais e efeitos deletérios causados pela própria doença.

Valenzuela et al.; (2003, p.72) expõe que fatores como "distúrbios gastrointestinais, fatores associados ao procedimento dialítico, acidose metabólica, distúrbios hormonais, doenças associadas ou intercorrentes”, também estão diretamente relacionados ao desenvolvimento da desnutrição em pacientes renais crônicos.

Os distúrbios hidroeletrolíticos citados por Favalessa et al.; (2009) são disfunções comuns da doença renal crônica bem como em pacientes em tratamento hemodialítico, condicionado em alterações substanciais, no metabolismo dos carboidratos, proteínas, lipídeos e micronutrientes, provocados por agentes nocivos encontrados nos fluidos sanguíneos do portador de doença renal.

A condição nutricional do paciente e o processo hemodialítico são com base nos escritos de Riella e Martins, (2001) os dois maiores fatores que influenciam no aumento da morbimortalidade, dessa forma, o trabalho de filtração realizado pelo dialisador, exerce efeitos significativos no tratamento, que refletem na sobrevida dos pacientes, portanto, é extremamente necessário que as máquinas realizem um processo eficaz e seguro. A autora ainda salienta que quando o processo de diálise 
ocorre de forma adequada é possível observar melhoras no quadro clínico dos pacientes tanto em relação, as complicações, referentes a tonturas, náuseas e vômitos quanto em relação à própria ingestão dietética.

Mahan; Scott-Stump, (2011) acrescentam que a atenção nutricional se mantém necessária para a intensificação do tratamento dialítico, sendo importante para a adequação e manutenção do estado nutricional e prevenção de complicações, devendo ser priorizada mesmo com o progresso de novos métodos de diálises e aperfeiçoamento de técnicas de transplante.

O planejamento alimentar do paciente renal crônico irá depender da condição em que este se encontra, portanto, devem ser observadas anteriormente, a causa base da doença, se hipertensão, diabetes, etc., o tratamento de substituição e conservação renal do qual o mesmo está sujeito, se hemodiálise ou diálise peritoneal, e os resultados dos exames laboratoriais de rotina que também são úteis para controlar de maneira integral a saúde dietética do paciente. Se porventura "a insuficiência renal piorar a dieta recomendada pode ser modificada" (Hospital Samaritano, 2014; National Kidney Foundation, 2007).

Os objetivos da terapia nutricional segundo Mahan; Escott-Stump, (2011) devem levar em consideração a recuperação, manutenção e alcance ideal do estado nutricional, o consumo controlado de líquidos, sódio e potássio, afim de que se evitem eventos de edema pela instabilidade eletrolítica, racionalização da ingestão de cálcio, fósforo e vitamina $\mathrm{D}$, para a prevenção de distúrbios minerais ósseos como a osteodistrofia renal e a manutenção e ajuste dos nutrientes energéticos, proteicos vitamínicos e minerálicos, promovendo dessa forma melhor qualidade de vida e tratamento ao paciente. Para alcançar esse propósito é de fundamental importância o conhecimento sobre o consumo de calorias, energia e certos nutrientes específicos como potássio e fósforo, salientando que esses nutrientes podem ter seu consumo restrito ou aumentado conforme a necessidade e as exigências do tratamento dialítico.

O tratamento dietético deve ser individualizado e adaptado conforme o progresso da doença, o tipo de terapia substitutiva e conforme a resposta do paciente. A adesão ou não da dieta é um grande desafio para a família e principalmente ao paciente, dessa forma ambos devem ser orientados. Com a necessidade de restrição de certos nutrientes é possível a recusa alimentar, promovendo assim a escolha de alimentos não recomendáveis (Marculino, 2004).

Ao iniciar o tratamento substitutivo, não só o paciente, mas toda sua família encontra dificuldades, relacionadas principalmente a alimentação, apesar de o consumo dietético ser complicado para o doente é importante que este siga as devidas recomendações para um bom andamento do tratamento. Segundo Mahan; Escott-Stump, (2011), o profissional nutricionista tem o papel de intervir em tais situações de forma positiva, utilizando-se de um bom planejamento alimentar individualizado, bem como a aplicação de atividades educativas e/ou orientações que advirta o paciente e o influencie de certa forma a seguir a alimentação proposta, pois como Cuppari, (2005), afirma muitos pacientes não seguem e/ou abandonam as orientações nutricionais, porque não entendem os motivos pelos quais devem priorizar uma alimentação restrita, assim como a relação que esta tem com o seu tratamento.

O tratamento individual de acordo com Vaconcellos, Braga e Soar, (2011) levam em consideração os aspectos socioeconômicos, psicológicos, culturais e habituais, devendo a dieta está adaptada ao doente e não o contrário.

Sabe-se que as necessidades nutricionais são modificadas nos pacientes renais crônicos que fazem tratamento hemodialítico, dessa forma, as quantidades exigidas de proteína, fósforo e potássio devem ser reajustadas, afim de sanar as perdas provocadas pelo tratamento de hemodiálise, onde o catabolismo é elevado, ou seja, o paciente perde muitos aminoácidos e peptídeos essências. Para o paciente em diálise é recomendado uma dieta hiperproteica de (1,2g/Kg/dia) de proteína ao dia, salientam também que além do reajuste na quantidade de proteína, deve-se atentar para as fontes desse macronutriente. Os valores recomendados são de $50 \%$ a $80 \%$ de proteínas de alto valor biológico (PAVB), afim de que seja garantido o aporte necessário de aminoácidos essenciais que o organismo necessita, esses valores são flexíveis, ou seja, a quantidade de proteína a ser consumida depende da necessidade desse paciente para mais ou para menos (Riella; Martins, 2001). 
O consumo proteico deve ser seguido criteriosamente visto que no processo de hemodiálise as perdas de proteínas nos pacientes são significativas, e tem como principal complicação a desnutrição que promove um maior atraso na alta clínica e aumento das taxas de mortalidade como já foi explicitado por (Berbel, 2011).

O potássio é um dos micronutrientes que deve ter o consumo controlado nos portadores de doença renal, visto que o metabolismo desse nutriente se encontra comprometido devido ao estado da doença, dessa forma os excessos desse mineral não são devidamente excretados. A principal reação envolvida com a quantidade elevada desse eletrólito no organismo do nefropata está relacionada à hipercalemia, que se caracteriza por altas taxas de potássio sérico que dentre outros sintomas suscita no aumento na incitabilidade nos sistemas cardíaco e nervoso, provocando alterações nos sentidos, como desmaio, dificuldades em respirar e em casos mais graves alterações nos batimentos cardíacos (Vaconcellos; Braga; Soar, 2011).

Cuppari, (2005) ressalta a informação de que o consumo de potássio deve ser cuidadosamente inspecionado pelo paciente renal, mesmo quando o mesmo não apresenta hipercalemia. Riella; Martins, (2001), acrescentam que o consumo de potássio deve ser de acordo com a capacidade excretória de urina, nesse sentido, para pacientes com "volume urinário" menor que $1000 \mathrm{ml}$ por dia, a recomendação individualizada diária correspondente a 1 e 3g.

Existem formas básicas para retirar o excesso desse micronutriente na hora do preparo dos alimentos, segundo orientações dietéticas medidas simples como descascar as frutas, legumes e feculentos, cortá-los e enxagua-los, coloca-los sobre fervura, torna possível a retirada de 50\% a 60\% do acumulo de potássio nos alimentos (Hospital Samaritano, 2014).

Riella; Martins, (2001) comentam que em relação ao consumo do mineral fósforo, o cuidado é um pouco maior, tendo em vista que o processo dialítico não é totalmente eficaz para sua eliminação no organismo, sendo necessário que o paciente faça uso de quelantes de fósforo, afim de que se diminua o efeito adverso desse nutriente. Como principais problemas do excesso de fósforo podem-se citar a hiperfosfatemia. Nerbass, (2010) comentou a associação entre a hiperfosfatemia e complicações cardiovasculares, que é a principal causa de morte em portadores de doença renal que fazem tratamento de diálise além do que, se relaciona a um severo risco para doença óssea, de regularização da glândula tireoide, provocando hiperparatireoidismo secundário pelo aumento do PTH (Canto, 2009).

Como característica da doença renal, o rim não consegue exercer sua funcionalidade, ou seja, processos reguladores do fósforo sérico como ultrafiltração e reabsorção estão prejudicados, esse mecanismo regulariza a quantidade do mineral no organismo, até que essas funções apresentem problemas, o organismo desenvolve maneiras de adaptação como o aumento na produção do paratormônio. A função do PTH está relacionada à regulação da quantidade de cálcio e fósforo, ou seja, o paratormônio age no organismo promovendo o aumento da captação de cálcio nos ossos, aumentando a eliminação do fósforo e estimulando a produção de vitamina D ativa pelos rins (MOE, 2001).

Pinheiro, (2014) esclarece que com a produção ineficaz de vitamina D e com a grande quantidade de fósforo sem ser excretada pela condição da doença renal, a glândula paratireoide é constantemente estimulada, afim de aumentar a excreção de fósforo, porém esse processo não ocorre, e a ação do hormônio se dá no aumento da captação de cálcio no ossos, que resulta em desmineralização óssea, e hiperparatireoidismo.

Sabendo-se disso, Cuppari, (2005) escreveu sobre o cuidado no consumo de alimentos fontes de fósforo por pacientes renais, havendo necessidade de monitorar o fósforo sérico e dietético, a indicação de consumo desse micronutriente deve ser baixa. A quantidade de fósforo diária deve ser flexível e o ajuste dessa quantidade na dieta deve ser realizada por profissionais devidamente capacitados, os valores indicados giram em torno de 800 a $1000 \mathrm{mg} / \mathrm{dia}$, sempre conforme a necessidade individual do paciente. 


\section{Conclusão}

De acordo com o estudo foi observado, que os portadores de doença renal crônica em hemodiálise são um público vulnerável, sendo necessário o constante acompanhamento do estado nutricional por ser um forte preditor de morbidades e mortalidades. Não há um método padrão ideal para se avaliar o estado nutricional, os mais utilizados são os métodos objetivos associados aos parâmetros bioquímicos.

\section{Referências}

Acuña, K., \& Cruz, T. Avaliação do estado nutricional de adultos e idosos e situação nutricional da população brasileira. Arq. Bras. Endocrinol. Metab. 48(3), 345- 361 .

Antczak, S. E. et al. Fisiopatologia básica. Guanabara Koogan.

Barbosa, L. M. M., Junior, M. P. A., \& Bastos, K. A. Preditores de qualidade de vida em pacientes com doença renal crônica em hemodiálise. J. Bras. Nefrol., 29(4), 222-229.

Bastos, M. G. et al. Doença Renal Crônica: Problemas e Soluções. J. Bras. Nefrol. 26(4), 202-215.

Bastos, M.G., Bregman, R., \& Kirsztajn, G. M. Doença renal crônica: frequente e grave, mas também prevenível e tratável. Rev. Assoc. Med. Bras., São Paulo, 56(2), 248-253.

Bastos, M. G., \& Kirsztajn, G. M. Doença renal crônica: importância do diagnóstico precoce, encaminhamento imediato e abordagem interdisciplinar estruturada para melhora do desfecho em pacientes ainda não submetidos à diálise. J. Bras. Nefrol. 33(1), 93-108.

Berbel, M. N. et al. Aspectos nutricionais na lesão renal aguda. Rev. Assoc. Med. Bras., 57(5), 600-606.

Blackburn, G. L. \& Thornton, P. A. Nutritional Assessment of the Hospitalized Patient. Med Clin North Am., 63(111), 03-15.

Brasil. Ministério da Saúde. Cadernos de Atenção Básica, Brasília, DF, 2006.

Brasil. Ministério da Saúde. Secretaria de Atenção à Saúde. Departamento de Atenção Básica. Orientações para a coleta e análise de dados antropométricos em serviços de saúde: Norma Técnica do Sistema de Vigilância Alimentar e Nutricional - SISVAN. Brasília, 2011.

Bueno, A. L., \& Czepielewski, M. A. O recordatório de 24 horas como instrumento na avaliação do consumo alimentar de cálcio, fósforo e vitamina D em crianças e adolescentes de baixa estatura. Rev. Nutr. 23(1), 65-73.

Cabral, P.C., Diniz, A.S., \& Arruda, I.K.G. Avaliação nutricional de pacientes em hemodiálise. Rev Nutr. 18(1), 29-40.

Calado, I. L. et al. Avaliação Nutricional de Pacientes Renais em Programa de Hemodiálise em um Hospital Universitário de São Luís do Maranhão. J. Bras. Nefrol., 29(4), 215-221.

Calado, I. L. et al. Diagnóstico nutricional de pacientes em hemodiálise na cidade de São Luís (MA). J. Bras. Nefrol., 22(5), 687-696.

Castro, M. C. M. Atualização em dialise: complicações agudas em hemodiálise. J. Bras. Nefrol., 23(2), 108-113.

Cavalcanti, C. T. A. Nível de atividade física e sintomas depressivos em pacientes submetidos à hemodiálise: um estudo de corte transversal. Fisioter Pesq., Recife, 21(2), 161-166.

Chaves G. A., Graça M. D., \& Gallo V. C. Consumo alimentar e estado nutricional de pacientes com doença renal crônica em tratamento dialítico. Rev. Bras. Ciênc. Saúde, 5(14), 11-17.

Cherchiglia, M. L. et al. Perfil epidemiológico dos pacientes em terapia renal substitutiva no Brasil, 2000-2004. Rev. Saúde Pública, 44(4), 639-49.

Cunha, M. S. et al. Avaliação da capacidade funcional e da qualidade de vida em pacientes renais crônicos submetidos a tratamento hemodialítico. Fisioter Pesq., 16(2), 155-60.

Cuppari, L. Nutrição Clínica no Adulto: Guia de medicina ambulatorial e hospitalar Unifesp-Escola Paulista de Medicina. (2a ed.), Manole.

Cuppari, L. Nutrição: nas doenças crônicas não-transmissíveis. Manoele.

Cuppari, L. Avesani, C. M. \& Kamimura, M. A. Nutrição na doença renal crônica. Manole.

Dutra, M. C. et al. Avaliação da função renal em idosos: um estudo de base populacional. J. Bras. Nefrol., 36(3), 297-303.

Dyniewicz, A. M. Metodologia da Pesquisa em saúde para iniciantes. (2a ed.),

Eaton, D. C., \& Pooler, J. P. Fisiologia Renal de Vander.(6a ed.), Artmed.

Favalessa, E. et al. Avaliação Nutricional e Consumo Alimentar de Pacientes com Insuficiência Renal Crônica. Revista Brasileira de Pesquisa em Saúde, 11(4), 39-48. 
Fernandes, B.R.M., \& Marshall, N.G. Avaliação nutricional de pacientes em hemodiálise: concordância entre métodos. Com. Ciências Saúde. 24(1), 39-50.

Fisberg, R. M., Marchioni, D. M. L., \& Colucci, A. C. A. Avaliação do consumo alimentar e da ingestão de nutrientes na prática clínica. Revista Arq. Bras. Endocrinol. Metab., 53(5), 617-624.

Freitas, A. T. V. S. et al. Prevalência e fatores associados à obesidade abdominal em pacientes em hemodiálise em Goiânia - GO. J. Bras. Nefrol., 35(4), 265272 .

Gomes, R., \& Nascimento, E.F. A produção do conhecimento da saúde pública sobre a relação homem-saúde: uma revisão bibliográfica. Cad. Saúde Pública. 22(5), 901-911.

Hall, J. E. Tratado de Fisiologia Médica. (12a ed.), Elsevier.

Helou, C. M. B. Potássio e Bicarbonato. J. Bras. Nefrol., 16(3), 22-25.

Hochman, B. et al. Desenhos de pesquisa. Acta Cir. Bras., 20(2), 02-09.

Instituto Brasileiro De Geografia E Estatística. Cidades. <http://www.cidades.ibge.gov.br/painel/painel.php?lang=\&codmun=210820\&search=\%7 C\%7Cinfogr\%E1ficos:-dados-gerais-do-munic\%EDpio>

Javera, V. M., \& Salado, G. A. Orientações nutricionais para pacientes em programa de hemodiálise. Revista Saúde e Pesquisa, 1(3), 319-324.

Kamimura, M. A. et al. Comparison of skinfold thicknessesand bioelectrical impedance analysis with dual-energy X-ray absorptiometry for the assessment of body fat in patients on longterm hemodialysis therapy. Nephrol dial transplant., 18(1), 101-105.

Lipschitz, D. A. Screening for nutritional status in the elderly. Prim Car., (21), 55-67.

Louvison, M. C. P. et al. Prevalência de pacientes em terapia renal substitutiva no Estado de São Paulo. Boletim Epidemiológico Paulista, 8(95), $23-42$.

Mahan, L. K., Scott-\& Stump, S. Krause: Alimentos, nutrição e dietoterapia. (12a ed.), Elsevier.

Malhotra, N. K. Pesquisa de Marketing. (4a ed.), Bookman.

Marculino, A. Q. Avaliação nutricional do paciente submetido à hemodiálise. 2004. 84 f. Trabalho de Conclusão de Curso (Pós Graduação em Nutrição) Universidade do Extremo Sul Catarinense - UNESC.

Martins, M. R. I., \& Cesarino, C. B. Qualidade de vida de pessoas com doença renal crônica em tratamento hemodialítico. Rev. Latino-am enfermagem., 13(5), $670-676$.

Matos, J. P. et al. Avaliação da sobrevida de cinco anos em hemodiálise no Brasil: uma coorte de 3.082 pacientes incidentes. J. Bras. Nefrol., 33(4), 436-41.

Moe, S. M. Equilíbrio entre fosforo e cálcio na insuficiência renal crônica: implicações e tratamento. <www.transdoreso.org/pdf/fosforol.pdf>.

Moreira, H. G., et al. Diabetes mellitus, hipertensão arterial e doença renal crônica: estratégias terapêuticas e suas limitações. Rev Bras Hipertens., 15(2), 111116.

Motta, V.T.Bioquímica Clínica para o Laboratório: Princípios e Interpretações. (5a ed.), Medbook.

Moura, L. et al. Monitoramento da doença renal crônica terminal pelo subsistema de Autorização de Procedimentos de Alta Complexidade - Apac - Brasil, 2000 a 2006. Epidemiol. Serv. Saúde, 18(2), 121-131.

Moura, L. R. R. Doenças Comuns: Insuficiência Renal. <http://www.sbn.org.br/publico/insuficiencia-renal>.

Moura, L. R. R. Hemodialise. <http://www.sbn.org.br/publico/hemodialise.>

National Kidney Foundation. Nutrição e Insuficiência Renal Crônica.

Nascimento, C. D., Moura, Marques, I. R. Intervenções de enfermagem nas complicações mais frequentes durante a sessão de hemodiálise: revisão da literatura. Rev. Bras. Enferm., 58(6), 719-722.

Nerbass, F. B. et al. Adesão e conhecimento sobre o tratamento de hiperfosfatemia de pacientes hiperfosfatemicos em hemodiálise. J. Bras. Nefrol., 32(2), $149-155$.

Nunes, M. B. et al. Perfil epidemiológico de pacientes renais crônicos em programa dialítico. Rev. Enfermagem UFPE on line, 8(1), 69-76.

Oliveira, L. M. C., Kubrusly, M., Mota, R. S., Silva, A. B., Moura, Oliveira, V. N. Desnutrição na insuficiência renal crônica: qual o melhor método diagnóstico na prática clínica?. J. Bras. Nefrol., 32(1), 57-70.

OMS- Organização Mundial de Saúde (WHO). World Health Organization. Physical Status: The use and interpretation of anthopometriy. Genebra: World Health Organization, 1995.

OSBORN, George. Orientações dietéticas gerais para o paciente renal crônico. <www.samaritano.org.br/pt.../orientacoes_dieteticas_paciente_renal.pdf>.

Pinheiro, P. Insuficiência renal, fósforo, pth e doença óssea,. < http://www.mdsaude.com/2010/03/insuficiencia-renal-fosforo-pth-doenca.html>. 
Pinto, D. E. et al. Associações entre ingestão energética, proteica e de fósforo em pacientes portadores de doença renal crônica em tratamento hemodialítico. $J$. Bras. Nefrol., 31(4), 269-276.

Ribeiro, R. C. H. M. et al. Depressão em idosos portadores de insuficiência renal crônica em tratamento hemodialitico. Acta Paul Enferm, 22, 505-508.

Riella, M. C., \& Moura, Martins, C. Nutrição e o rim. Guanabara Koogan.

Romão Junior, J. E. et al. Alterações de cálcio e fósforo séricos e hiperparatireoidismo na insuficiência renal crônica incidente. J. Bras. Nefrol., 16(1), 6-11.

Romão-Júnior, J. E. A doença renal crônica: do diagnóstico ao tratamento. Prática hospitalar. J. Bras. Nefrol., 52(15), 183-187.

Romão-Júnior, J. E. Doença Renal Crônica: Definição, Epidemiologia e Classificação. J. Bras. Nefrol. 26(3), 1-3.

Sá, J. R. Diabetes e doença renal crônica. 2014. <http://www.diabetes.org.br/artigos-sobre-diabetes/diabetes-e-doenca-renal-cronica>.

Santos, A. C. B. et al. Associação entre qualidade de vida e estado nutricional em pacientes renais crônicos em hemodiálise. J. Bras. Nefrol., 35(4), $279-288$.

Scagliusi, F.B., Lancha Júnior, A.H. Subnotificação da ingestão energética na avaliação do consumo alimentar. Rev Nutr., 16(4), 471-81,

Sesso, R.C.C., \& Gordan, P., Dados disponíveis sobre a doença renal crônica no Brasil. J. Bras. de Nefrologia, 29, 9-12.

Sesso, R. C. et al. Relatório do Censo Brasileiro de Diálise Crônica 2012. J. Bras. Nefrol., 36(1), 48-53.

Sesso, R.C. Inquérito epidemiológico em unidades de diálise no Brasil. J. Bras. Nefrol., 22, 23-26.

Soares, G. L. et al. Perfil epidemiológico de pacientes renais crônicos em tratamento hemodialítico: um estudo descritivo. Revista Multiprofissional em Saúde do Hospital São Marcos, 1(1), 01-08.

Sociedade Brasileira De Nefrologia (SBN). Perfil da doença renal crônica o desafio brasileiro 2007.

Sociedade Brasileira De Nefrologia (SBN). Doença Renal Crônica (Pré-terapia Renal Substitutiva): Diagnóstico.

Sociedade Brasileira De Nefrologia (SBN). Insuficiência renal aguda.

Sociedade Brasileira De Nefrologia (SBN). Censo de Dialise 2008.

Sociedade Brasileira De Nefrologia (SBN). Censo de Dialise 2013.

Sociedade Brasileira De Nefrologia (SBN). Hemodiálise.

Sociedade Brasileira De Nutrição Parenteral E Enteral/ Associação Brasileira De Nutrologia. Terapia Nutricional para Pacientes em Hemodiálise Crônica.

Sodré, F. L. et al. Avaliação da função e da lesão renal: um desafio laboratorial. J Bras. Patol. Med. Lab., 43(5), 329-337.

Sousa, K. T. et al. Baixo peso e dependência funcional em idosos institucionalizados de Uberlândia (MG), Brasil. Ciênc. saúde coletiva, 19(8), 3513- 3520.

Stefanelli, C. et al. Avaliação nutricional de pacientes em hemodiálise. J. Health Sci. Inst. 28(3), 268-271.

Szuster, D. A. C. et al. Sobrevida de pacientes em diálise no SUS no Brasil. Cad. Saúde Pública, 28(3), 415-424.

Tonelli, M., \& Riella, M. Doença renal crônica e o envelhecimento da população. J. Bras. Nefrol., 36(1), 1-5.

Universidade Católica De Brasília. Metodologia da pesquisa.

Valenzuela, R. G. V. et al. Estado nutricional de pacientes com insuficiência renal crônica em hemodiálise no Amazonas. Revista da Associação Médica Brasileira, 49(1), 72-78.

Vaconcellos, A. M. A., Braga, E. C. L., \& Soar, C. Efeito De Um Programa nutricional no controle da hipercalemia em pacientes com Insuficiência Renal Crônica. In: XIV Encontro Latino Americano de Iniciação Científica e X

Zambra, B., \& Huth, A. Terapia nutricional em pacientes portadores de insuficiência renal crônica em hemodiálise. Revista contexto \& saúde. 10(19), 67-72. 